\title{
Bruhat order, rationally smooth Schubert varieties, and hyperplane arrangements
}

\author{
Suho Oh"1 and Hwanchul Yoo
}

Department of Mathematics, Massachusetts Institute of Technology, 77 Massachusetts Ave, Cambridge, MA 02139

\begin{abstract}
We link Schubert varieties in the generalized flag manifolds with hyperplane arrangements. For an element of a Weyl group, we construct a certain graphical hyperplane arrangement. We show that the generating function for regions of this arrangement coincides with the Poincaré polynomial of the corresponding Schubert variety if and only if the Schubert variety is rationally smooth.

Résumé. Nous relions des variétés de Schubert dans le variété flag généralisée avec des arrangements des hyperplans. Pour un élément dún groupe de Weyl, nous construisons un certain arrangement graphique des hyperplans. Nous montrons que la fonction génératrice pour les régions de cet arrangement coincide avec le polynome de Poincaré de la variété de Schubert correspondante si et seulement si la variété de Schubert est rationnellement lisse.
\end{abstract}

Keywords: Bruhat order, Schubert Variety, Rational Smoothness, Palidromic, Hyperplanes, Coxeter arrangement

\section{Introduction}

For an element of a Weyl group $w \in W$, let $P_{w}(q):=\sum_{u \leq w} q^{\ell(u)}$, where the sum is over all elements $u \in W$ below $w$ in the (strong) Bruhat order. Geometrically, the polynomial $P_{w}(q)$ is the Poincare polynomial of the Schubert variety $X_{w}=B w B / B$ in the flag manifold $G / B$.

The inversion hyperplane arrangement $\mathcal{A}_{w}$ is defined as the collection of hyperplanes corresponding to all inversions of $w$. Let $R_{w}(q):=\sum_{r} q^{d\left(r_{0}, r\right)}$ be the generating function that counts regions $r$ of the arrangement $\mathcal{A}_{w}$ according to the distance $d\left(r_{0}, r\right)$ from the fixed initial region $r_{0}$.

The main result of the paper is the claim that $P_{w}(q)=R_{w}(q)$ if and only if the Schubert variety $X_{w}$ is rationally smooth. We have previously given an elementary combinatorial proof for Type A case of this problem in Oh et al. (2008).

According to the criterion of Peterson and Carrell (1994), the Schubert variety $X_{w}$ is rationally smooth if and only if the Poincare polynomial $P_{w}(q)$ is palindromic, that is $P_{w}(q)=q^{\ell(w)} P_{w}\left(q^{-1}\right)$. If $w$ is not rationally smooth then the polynomial $P_{w}(q)$ is not palindromic, but the polynomial $R_{w}(q)$ is always palindromic. So $P_{w}(q) \neq R_{w}(q)$ in this case. Hence it is enough to show that $P_{w}(q)=R_{w}(q)$ when $w$ is rationally smooth. Our proof is purely combinatorial, combining basics of Weyl groups with a result from Billey and Postnikov (2005).

${ }^{\dagger}$ S.O. was supported in part by Samsung Scholarship.

1365-8050 (c) 2010 Discrete Mathematics and Theoretical Computer Science (DMTCS), Nancy, France 


\section{Rational smoothness of Schubert varieties and Inversion hy- perplane arrangement}

In this section we will explain how rational smoothness can be expressed by conditions on the lower Bruhat interval. We will also define the Inversion hyperplane arrangement. In this paper, unless stated otherwise, we refer to the strong Bruhat order.

Let $G$ be a semisimple simply-connected complex Lie group, $B$ a Borel subgroup and $\mathfrak{h}$ the corresponding Cartan subalgebra. Let $W$ be the corresponding Weyl group, $\Delta \subset \mathfrak{h}^{*}$ be the set of roots and $\Pi \subset \Delta$ be the set of simple roots. The choice of simple roots determines the set of positive roots. We will write $\alpha>0$ for $\alpha \in \Delta$ being a positive root. Following the conventions of Björner and Brenti (2005), let $S$ be the set of simple reflections and $T:=\left\{w s w^{-1}: s \in S, w \in W\right\}$ be the set of reflections. Set $\Pi=\left\{\alpha_{1}, \cdots, \alpha_{n}\right\}, S=\left\{s_{1}, \cdots, s_{n}\right\}$ and index them properly so that $s_{i}$ and $\alpha_{i}$ corresponds to the same node of the Dynkin diagram for $1 \leq i \leq n$. Then there is a bijection between $T$ and $\Delta$ by matching $w s_{i} w^{-1}$ with $w\left(\alpha_{i}\right)$. Then $w s_{i} w^{-1}$ is exactly the reflection that reflects by the hyperplane corresponding to the root $w\left(\alpha_{i}\right)$.

We have the following definitions as in Björner and Brenti (2005):

$$
\begin{gathered}
T_{L}(w):=\{t \in T: \ell(t w)<\ell(w)\}, \\
T_{R}(w):=\{t \in T: \ell(w t)<\ell(w)\}, \\
D_{L}(w):=T_{L}(w) \cap S, \\
D_{R}(w):=T_{R}(w) \cap S .
\end{gathered}
$$

They are called the left(right) associated reflections of $w$ and left(right) descent set of $w$. In this paper, we concentrate on lower Bruhat intervals in $W$, [id, $w]:=\left\{u \in S_{n} \mid u \leq w\right\}$. They are related to Schubert varieties $X_{w}=\overline{B w B / B}$ inside the generalized flag manifold $G / B$. The Poincaré polynomial of the Schubert variety $X_{w}$ is the rank generating function for the interval [id, $w$ ], e.g., see Billey et al. (2000):

$$
P_{w}(q)=\sum_{u \leq w} q^{\ell(u)} .
$$

For convenience, we will say that $P_{w}(q)$ is the Poincare polynomial of $w$. And we will say that $w$ is rationally smooth if $X_{w}$ is rationally smooth. Due to Carrell and Peterson, one can check whether the rational locus of a Schubert variety is smooth or not by studying $P_{w}(q)$. Let us denote a polynomial $f(q)=a_{0}+a_{1} q+\cdots+a_{d} q^{d}$ as palindromic if $f(q)=q^{d} f\left(q^{-1}\right)$, i.e., $a_{i}=a_{d-i}$ for $i=0, \ldots, d$.

Theorem 1 (Carrell-Peterson Carrell (1994), see also (Billey et al. 2000, Sect. 6.2)) For any element of a Weyl group $w \in W$, the Schubert variety $X_{w}$ is rationally smooth if and only if the Poincaré polynomial $P_{w}(q)$ is palindromic.

For each $w \in W$, we will be comparing this polynomial $P_{w}(q)$ with another polynomial, that comes from an associated hyperplane arrangement. To assign a hyperplane arrangement to each $w \in W$, we first need the definition of the inversion set of $w$. The inversion set $\Delta_{w}$ of $w$ is defined as the following:

$$
\Delta_{w}:=\{\alpha \mid \alpha \in \Delta, \alpha>0, w(\alpha)<0\} .
$$


For type A case, this gives the usual definition of an inversion set for permutations. Let us define the arrangement $\mathcal{A}_{w}$ as the collection of hyperplanes $\alpha(x)=0$ for all roots $\alpha \in \Delta_{w}$. Let $r_{0}$ be the fundamental chamber of $\mathcal{A}_{w}$, the chamber that contains the points satisfying $\alpha(x)>0$ for all $\alpha \in \Delta_{w}$. Then we can define a polynomial from this $\mathcal{A}_{w}$ :

$$
R_{w}(q):=\sum_{r} q^{d\left(r_{0}, r\right)},
$$

where the sum is over all chambers of the arrangement $\mathcal{A}_{w}$ and $d\left(r_{0}, r\right)$ is the number of hyperplanes separating $r_{0}$ and $r$. Our goal in this paper is to show that $R_{w}(q)=P_{w}(q)$ whenever $P_{w}(q)$ is palindromic.

Remark 2 We have $P_{w}(q)=P_{w^{-1}}(q)$ and $R_{w}(q)=R_{w^{-1}}(q)$ by definition. Whenever we use this fact, we will call this the duality of $P_{w}(q)$ and $R_{w}(q)$.

Given an arrangement $\mathcal{A}_{w}$ and its subarrangement $\mathcal{A}^{\prime}$, let $c$ be a chamber of $\mathcal{A}^{\prime}$. Then a chamber graph of $c$ with respect to $\mathcal{A}_{w}$ is defined as a directed graph $G=(V, E)$ where

- The vertex set $V$ consists of vertices representing each chambers of $\mathcal{A}_{w}$ contained in $c$,

- we have an edge directed from vertex representing chamber $c_{1}$ to a vertex representing chamber $c_{2}$ if $c_{1}$ and $c_{2}$ are adjacent and $d\left(r_{0}, c_{1}\right)+1=d\left(r_{0}, c_{2}\right)$.

We will say that $\mathcal{A}_{w}$ is uniform with respect to $\mathcal{A}^{\prime}$ if for all chambers of $\mathcal{A}^{\prime}$, chamber graphs with respect to $\mathcal{A}_{w}$ are isomorphic. One can easily see that if $\mathcal{A}_{u}$ is a subarrangement of $\mathcal{A}_{w}$ and $\mathcal{A}_{w}$ is uniform with respect to $\mathcal{A}_{u}$, then $R_{w}(q)$ is divided by $R_{u}(q)$.

\section{Parabolic Decomposition}

In this section, we introduce a theorem of Billey and Postnikov (2005) regarding parabolic decomposition that will serve as a key tool in our proof. Let's first recall the definition of the parabolic decomposition. Given a Weyl group $W$, fix a subset $J$ of simple roots. Denote $W_{J}$ to be the parabolic subgroup generated by simple reflections of $J$. Let $W^{J}$ be the set of minimal length coset representatives of $W_{J} \backslash W$. Then it is a well-known fact that every $w \in W$ has a unique parabolic decomposition $w=u v$ where $u \in W_{J}, v \in$ $W^{J}$ and $\ell(w)=\ell(u)+\ell(v)$.

Lemma 3 (van den Hombergh (1974)) For any $w \in W$ and subset $J$ of simple roots, $W_{J}$ has a unique maximal element below $w$.

We will denote the maximal element of $W_{J}$ below $w$ as $m(w, J)$.

Theorem 4 (Billey and Postnikov (2005)) Let $J$ be any subset of simple roots. Assume $w \in W$ has parabolic decomposition $w=u v$ with $u \in W_{J}$ and $v \in W^{J}$ and furthermore, $u=m(w, J)$. Then

$$
P_{w}(t)=P_{u}(t) P_{v}^{W^{J}}(t)
$$

where $P_{v}^{W^{J}}=\sum_{z \in W^{J}, z \leq v} t^{\ell(z)}$ is the Poincaré polynomial for $v$ in the quotient.

This decomposition is very useful in the sense that it allows us to factor the Poincare polynomials. We will say that $J=\Pi \backslash\{\alpha\}$ is leaf-removed if $\alpha$ corresponds to a leaf in the Dynkin diagram of $\Pi$.

The following theorem of Billey and Postnikov (2005) tells us that we only need to look at maximal leaf-removed parabolic subgroups for our purpose. 
Theorem 5 (Billey and Postnikov (2005)) Let $w \in W$ be a rationally smooth element. Then there exists a maximal proper subset $J=\Pi \backslash\{\alpha\}$ of simple roots, such that

1. we have a decomposition of $w$ or $w^{-1}$ as in Theorem 4 .

2. $\alpha$ corresponds to a leaf in the Dynkin diagram of $W$.

We will call the parabolic decompositions that satisfies the conditions of the above theorem as $\boldsymbol{B P}$ decompositions. For Weyl groups of type A,B and D, there is a stronger result by Billey:

Lemma 6 (Billey (1998)) Let $W$ be a Weyl group of type A,B or D. Let $w \in W$ be a rationally smooth element. If $w$ is not the longest element of $W$, then there exists a BP-decomposition of $w$ or $w^{-1}$ with respect to $J$ such that $P^{J}(v)$ is of the form $q^{l}+q^{l-1}+\cdots+q+1$, where l is the length of $v$.

If $v$ satisfies the conditions of the above lemma, we will say that $v$ is a chain element of $W^{J}$. Using the fact that Dynkin diagrams of type A or D are simply-laced, it is easy to deduce the following result from the above lemma.

Corollary 7 Let $W$ be a Weyl group of type A or D. If $w \in W$ is rationally smooth then there exists a BP-decomposition of $w$ or $w^{-1}$ with respect to $J=\Pi \backslash\{\alpha\}$ such that $v$ is the longest element of $W_{I}^{I \cap J}$ for some $I \subset \Pi$ containing $\alpha$.

Using computers, we have found a nice property of palindromic intervals in maximal parabolic quotient groups of type E.

Proposition 8 Let $W$ be a Weyl group of type A,D and $E$ and let $J=\Pi \backslash\{\alpha\}$, where $\alpha$ corresponds to a leaf in the Dynkin diagram. Then, $v$ has palindromic lower interval in $W^{J}$ if and only if there exists a subset I of $\Pi$ containing $\alpha$ such that $v$ is the longest element in $W_{I}^{I \cap J}$.

Let's look at an example. Choose $D_{6}$ to be our choice of Weyl group and label the simple roots $\Pi=\left\{\alpha_{1}, \cdots, \alpha_{6}\right\}$ so that the labels match the corresponding nodes in the Dynkin diagram 1 If we set $J=\Pi \backslash\left\{\alpha_{1}\right\}$, then the list of $v \in W^{J}$ such that the lower interval in $W^{J}$ being palindromic is:

$$
i d, s_{1}, s_{1} s_{2}, s_{1} s_{2} s_{3}, s_{1} s_{2} s_{3} s_{4}, s_{1} s_{2} s_{3} s_{4} s_{5}, s_{1} s_{2} s_{3} s_{4} s_{6}, s_{1} s_{2} s_{3} s_{4} s_{5} s_{6} s_{4} s_{3} s_{2} s_{1} .
$$

Each of them are the longest elements of $W_{I}^{I \cap J}$, where $I$ is the set of simple reflections appearing in $v$. One can see that the set of nodes $I$ is connected inside the Dynkin diagram of $D_{6}$.

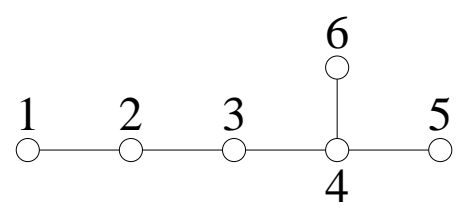

Fig. 1: Dynkin diagram of $D_{6}$

Now we will study how $R_{w}(q)$ behaves with respect to the BP-decomposition . Using the notations of Proposition 8 , our first step is to prove that every reflection formed by simple reflections in $I \cap J$ is in $T_{R}(u)$. We need the following lemma to prove it: 
Lemma 9 Let $w \in W$ be a rationally smooth element and $w=u v$ be a BP-decomposition. Then every simple reflection in $J$ appearing in the reduced word of $v$ is a right descent of $u$.

Proof: Multiplying $t \in T_{L}(w)$ to $w$ corresponds to deleting one simple reflection in a certain reduced word of $w$. If we delete every simple reflection appearing in $v$ but one in $J$, then the resulting element is in $W_{J}$ and is below $w$. Hence by maximality of $u$, it is below $u$.

Actually, we can state much more about $u$ in terms of simple reflections of $J$ appearing in $v$.

Lemma 10 Let $w=$ uv be a BP-decomposition with respect to $J$. Let I be the subset of $\Pi$ that appears in the reduced word of $v$. Then every reflection formed by simple reflections in $I \cap J$ is a right inversion reflection of $u$. In fact, there is a minimal length decomposition $u=u^{\prime} u_{I \cap J}$ where $u_{I \cap J}$ is the longest element of $W_{I \cap J}$.

Proof: Take the parabolic decomposition of $u$ under the right quotient by $W_{I \cap J}$. Say, $u=u^{\prime} u_{I \cap J}$. Then $u^{\prime}$ is the minimal length representative of $u$ in $W / W_{I \cap J}$. For any simple reflection $s \in I \cap J$, the minimal length representative of $u s$ in $W / W_{I \cap J}$ is still $u^{\prime}$, hence the parabolic decomposition of $u s$ is $u s=u^{\prime}\left(u_{I \cap J} s\right)$. Since $s$ is a right descent of $u$ by Lemma 9 , $s$ is a right descent of $u_{I \cap J}$. Therefore $u_{I \cap J}$ is the longest element in $W_{I \cap J}$. The rest follows from this.

The above lemma tells us that for each rationally smooth $w \in W$, we can decompose $w$ or $w^{-1}$ to $u^{\prime} u_{I \cap J} v$ where $u v$ is the BP-decomposition with respect to $J, u=u^{\prime} u_{I \cap J}$ and $u_{I \cap J}$ is the longest element of $W_{I \cap J}$. Recall that we denote by $\Delta_{w}$ the inversion set of $w \in W$. For $I \subset \Pi$, we will denote $\Delta_{I}$ the set of roots of $W_{I}$. We have a decomposition

$$
\Delta_{w}=\Delta_{u^{\prime}} \sqcup u^{\prime} \Delta_{u_{I \cap J}} u^{\prime-1} \sqcup u \Delta_{v} u^{-1} .
$$

One can see that $\Delta_{u_{I \cap J}}=\Delta_{I \cap J}$ and $\Delta_{v} \subseteq \Delta_{I} \backslash \Delta_{I \cap J}$. And this tells us that $u^{\prime} \Delta_{u_{I \cap J}} u^{\prime-1}=$ $u \Delta_{I \cap J} u^{-1}$. By duality, let's assume we have decomposed some rationally smooth $w$ as above. Let $\mathcal{A}_{1}, \mathcal{A}_{0}, \mathcal{A}_{2}$ denote the hyperplane arrangement coming from $u^{-1} \Delta_{u^{\prime}} u, \Delta_{I \cap J}, \Delta_{v}$. We can study $\mathcal{A}:=$ $\mathcal{A}_{1} \sqcup \mathcal{A}_{0} \sqcup \mathcal{A}_{2}$ instead of looking at $\mathcal{A}_{w}$.

Lemma 11 Let c be some chamber inside $\mathcal{A}_{1} \sqcup \mathcal{A}_{0}$. Let $c^{\prime}$ be the chamber of $\mathcal{A}_{0}$ that contains $c$. Then the chamber graph of $c$ with respect to $\mathcal{A}$ is isomorphic to the chamber graph of $c^{\prime}$ with respect to $\mathcal{A}_{0} \sqcup \mathcal{A}_{2}$.

Proof: Let $c_{1}$ and $c_{2}$ be two different chambers of $\mathcal{A}$ contained in $c$. They are separated by a hyperplane in $\mathcal{A}_{2}$. Let $c_{1}^{\prime}\left(c_{2}^{\prime}\right)$ be the chamber of $\mathcal{A}_{0} \sqcup \mathcal{A}_{2}$ that contains $c_{1}\left(c_{2}\right)$. $c_{1}^{\prime}$ and $c_{2}^{\prime}$ are different chambers since they are separated by the hyperplane that separates $c_{1}$ and $c_{2}$. If $c_{1}$ and $c_{2}$ are adjacent, then $c_{1}^{\prime}$ and $c_{2}^{\prime}$ are adjacent. If $c_{1}^{\prime}$ and $c_{2}^{\prime}$ are adjacent but $c_{1}$ and $c_{2}$ are not, that means there is a hyperplane of $\mathcal{A}_{1}$ that separates $c_{1}$ and $c_{2}$. But that contradicts the fact that $c_{1}$ and $c_{2}$ are both contained in the same chamber of $\mathcal{A}_{1} \sqcup \mathcal{A}_{0}$. So $c_{1}$ and $c_{2}$ are adjacent if and only if $c_{1}^{\prime}$ and $c_{2}^{\prime}$ are. From the fact that the distance from the fundamental chamber is equal to the number of hyperplanes that separate the chamber from the fundamental chamber, we see that the direction of the corresponding edges in the chamber graphs are the same.

Hence it is enough to show that the number of chambers of $\mathcal{A}$ in $c$ equals number of chambers of $\mathcal{A}_{0} \sqcup \mathcal{A}_{2}$ in $c^{\prime}$. And this follows from showing that any chamber of $\mathcal{A}_{0} \sqcup \mathcal{A}_{2}$ shares a common interior 
point with a chamber of $\mathcal{A}_{0} \sqcup \mathcal{A}_{1}$ as long as they are contained in the same chamber of $\mathcal{A}_{0}$. To show this, we may include additional hyperplanes to $\mathcal{A}_{1}$ and $\mathcal{A}_{2}$. So let $\mathcal{A}_{2}$ be the hyperplane arrangement of $\Delta_{I} \backslash \Delta_{I \cap J}$ and $\mathcal{A}_{1}$ hyperplane arrangement of $\Delta \backslash \Delta_{I}$. Now $\mathcal{A}$ is just the Coxeter arrangement of $W$, and each chamber of $\mathcal{A}$ is indexed by $w \in W$.

We have a parabolic decomposition of $W$ by $W_{I \cap J} W_{I}^{I \cap J} W^{I}$. Fixing a chamber $c$ of $\mathcal{A}_{0}$ corresponds to fixing an element of $W_{I \cap J}$. In $c$, fixing a chamber $x(y)$ of $\mathcal{A}_{0} \sqcup \mathcal{A}_{2}\left(\mathcal{A}_{0} \sqcup \mathcal{A}_{1}\right)$ corresponds to fixing an element of $W_{I}^{I \cap J}\left(W^{I}\right)$. So given any such chamber $x$ and $y$, we can find a chamber of $\mathcal{A}$ contained in them. This concludes the argument.

Corollary 12 In the above decomposition, if $\mathcal{A}_{w^{\prime}}$ is uniform with respect to $\mathcal{A}_{u}$ and $v$ is the longest element of $W_{I}^{J}$, then $R_{u}(q)=P_{u}(q)$ implies $R_{w}(q)=P_{w}(q)$.

Proof: If $v$ is the longest element of $W_{I}^{J}$, then $w^{\prime}:=u_{I \cap J} v$ is the longest element of $W_{I}$. Then it is obvious that $\mathcal{A}_{w^{\prime}}$ is uniform with respect to $\mathcal{A}_{u}$. Now it follows from above lemma that $R_{w}(q) / R_{u}(q)=$ $R_{u_{I \cap J} v}(q) / R_{u_{I \cap J}}(q)$. Since we also know that the right hand side equals $P_{v}^{W^{J}}(q), R_{u}(q)=P_{u}(q)$ implies $R_{w}(q)=P_{w}(q)$.

In the next section, we will use the above lemma and corollary to prove the main theorem for type $\mathrm{A}, \mathrm{B}, \mathrm{D}$ and $\mathrm{E}$.

\section{The main Proof}

In this section, we prove the main theorem. Type $\mathrm{G}$ case is trivial and omitted, type $\mathrm{F}$ case is done with a computer and is omitted in this extended abstract. For type A,D and E, the proof is very easy using Proposition 8 and Corollary 12 .

Proposition 13 Let $W$ be a Weyl group of type A,D or E. Let $w$ be a rationally smooth element. Then $R_{w}(q)=P_{w}(q)$.

Proof: Decompose $w$ or $w^{-1}$ as in the remark preceding Lemma 11 By applying Proposition 8 , we see that $v$ is the longest element of $W_{I}^{J}$. Now we can apply Corollary 12 So we can replace $w$ with some rationally smooth $u$ that is contained in some Weyl group of type A,D or E with strictly smaller rank. Now the result follows from an obvious induction argument.

For type B, we will use Lemma 6 and Lemma 11 . Let's denote $\Pi=\left\{\alpha_{0}=x_{1}, \alpha_{1}=x_{2}-x_{1}, \cdots, \alpha_{n}=\right.$ $\left.x_{n+1}-x_{n}\right\}$. We will be studying $W^{\Pi \backslash\left\{\alpha_{0}\right\}}$ and $W^{\Pi \backslash\left\{\alpha_{n}\right\}}$. In both of them, if in the reduced word of $v$ there is an adjacent commuting letters, then $v$ is not a chain element. So when $J=\Pi \backslash\left\{\alpha_{0}\right\}$, the chain elements are

$$
i d, s_{0}, s_{0} s_{1}, s_{0} s_{1} s_{2}, \cdots, s_{0} s_{1} \ldots s_{n}, s_{0} s_{1} s_{0} .
$$

And when $J=\Pi \backslash\left\{\alpha_{n}\right\}$, the chain elements are

$$
i d, s_{n}, s_{n} s_{n-1}, \cdots, s_{n} s_{n-1} \ldots s_{1} s_{0}, s_{n} s_{n-1} \ldots s_{1} s_{0} s_{1}, \ldots, s_{n} s_{n-1} \ldots s_{1} s_{0} s_{1} \ldots s_{n-1} s_{n} .
$$

Proposition 14 Let $W$ be a Weyl group of type B. Let $w$ be a rationally smooth element. Then $R_{w}(q)=$ $P_{w}(q)$. 
Proof: By Lemma 6, we may assume $w$ or $w^{-1}$ decomposes to $u v$ where $u \in W_{J}, v \in W^{J}, J$ is leafremoved and $v$ is a chain-element. Let's first show that when $u$ is the longest element of $W_{J}$, then $\mathcal{A}_{w}$ is uniform with respect to $\mathcal{A}_{u}$ and $R_{w}(q)=P_{w}(q)$. Instead of looking at hyperplane arrangement coming from $\Delta_{w}=\Delta_{u} \sqcup u \Delta_{v} u^{-1}$, we can look at the hyperplane arrangement coming from $u^{-1} \Delta_{w} u=\Delta_{u} \sqcup \Delta_{v}$. So $\mathcal{A}_{u}$ consists of hyperplanes coming from $\Delta_{u}$ and $\mathcal{A}_{v}$ consists of hyperplanes coming from $\Delta_{v}$.

When $J=\Pi \backslash\left\{\alpha_{0}\right\}$, we have $\Delta_{v} \subset\left\{x_{1}, \cdots, x_{n}\right\}$ and $\left|\Delta_{v}\right|=\ell(v)$. Choosing a chamber in $\mathcal{A}_{u}$ is equivalent to giving a total ordering on $\left\{x_{1}, \cdots, x_{n}\right\}$. Choosing a chamber in $\mathcal{A}_{v}$ is equivalent to assigning signs to roots of $\Delta_{v}$. Given any total ordering on $\left\{x_{1}, \cdots, x_{n}\right\}$, there is a unique way to assign $t$ number of +'s and $|v|-t$ number of -'s to $\Delta_{v}$ so that it is compatible with the total order on $\Delta_{v}$. This tells us that $\mathcal{A}_{w}$ is uniform with respect to $\mathcal{A}_{u}$ and $R_{w}(q)=R_{u}(q)\left(1+q+\cdots+q^{|v|}\right)=R_{u}(q) P_{v}^{W^{J}}(q)$. When $J=\Pi \backslash\left\{\alpha_{n}\right\}$, the proof is pretty much similar and is omitted.

Now let's return to the general case. Using Lemma 11 and above argument, we can replace $w$ with some rationally smooth $u$ that is contained in some Weyl group of type A or B with strictly smaller rank. Then the result follows from an obvious induction argument.

\section{Further remarks}

As in Oh et al. (2008), our proof of the main theorem is based on a recurrence relation. It would be interesting to give a proof based on a bijection between elements of $[i d, w]$ and regions of $\mathcal{A}_{w}$.

The statement of our main theorem can be extended to Coxeter groups. Although we don't have Schubert varieties for Coxeter groups, the Poincaré polynomial $P_{w}(q)$ can still be defined as the rank generating function of the interval $[i d, w]$.

Conjecture 15 Let $W$ be any Coxeter group. Then $[i d, w]$ is palindromic if and only if $P_{w}(q)=R_{w}(q)$.

Our proof for the Weyl group case relied heavily on Theorem 5 and Proposition 8 Described a bit roughly, the former helps us to find the recurrence for $P_{w}(q)$ and the latter helps us to find the recurrence for $R_{w}(q)$. So the key would be to extending these two statements. For Proposition 8, it is easy to see that one direction holds for all Weyl groups. We give a slightly weakened statement that seems to hold for all Weyl groups.

Conjecture 16 Let $W$ be a Weyl group and let $J$ be a maximal proper subset of the simple roots. Then, $v$ has palindromic lower interval in $W^{J}$ if and only if the interval is isomorphic to a maximal parabolic quotient of some Weyl group.

Let's look at an example for the above conjecture. Choose $F_{4}$ to be our choice of Weyl group and label the simple roots $\Pi=\left\{\alpha_{1}, \cdots, \alpha_{4}\right\}$ so that the labels match the corresponding nodes in the Dynkin diagram 2. If we set $J=\Pi \backslash\left\{\alpha_{4}\right\}$, then the list of $v \in W^{J}$ such that the lower interval in $W^{J}$ being palindromic is:

$$
i d, s_{4}, s_{4} s_{3}, s_{4} s_{3} s_{2}, s_{4} s_{3} s_{2} s_{1}, s_{4} s_{3} s_{2} s_{3}, s_{4} s_{3} s_{2} s_{3} s_{4}, s_{4} s_{3} s_{2} s_{3} s_{1} s_{2} s_{3} s_{4} s_{3} s_{2} s_{3} s_{1} s_{2} s_{3} s_{4}
$$

Those that do not correspond to longest elements of $W_{I}^{I \cap J}$ for some $I \subset \Pi$ are $s_{4} s_{3} s_{2}, s_{4} s_{3} s_{2} s_{3}$ and $s_{4} s_{3} s_{2} s_{1}$. But in these cases, hasse diagram of $[i d, v]$ in $W^{J}$ is a chain. So we can say that $[i d, v]$ in $W^{J}$ is isomorphic to a maximal parabolic quotient of a Weyl group of type $\mathrm{A}$ in these cases. 


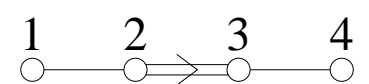

Fig. 2: Dynkin diagram of $F_{4}$

One nice property that $R_{w}(q)$ has is that it is always palindromic regarthless of the rational smoothness of $w$. And this is a property that intersection homology Poncaré polynomial $I P_{w}(q)$ also has.

So it would be interesting to compare these two polynomials.

\section{Acknowledgements}

We would like to thank Alexander Postnikov and Sara Billey for useful remarks.

\section{References}

S. C. Billey. Pattern avoidance and rational smoothness of schubert varieties. Adv. Math, 139:141-156, 1998.

S. C. Billey and A. Postnikov. Smoothness of schubert varieties via patterns in root subsystems. Advances in Applied Mathematics, 34:447-466, 2005.

S. C. Billey, , and V. Lakshmibai. Singular Loci of Schubert Varieties. Birkhäuser, 2000.

A. Björner and F. Brenti. Combinatorics of Coxeter Groups. Springer, 2005.

J. B. Carrell. The bruhat graph of a coxeter group, a conjecture of deodhar, and rational smoothness of schubert varieties. Proceedings of Symposia in Pure Math, 56:53-61, 1994.

S. Oh, A. Postnikov, and H. Yoo. Bruhat order, smooth schubert varieties, and hyperplane arrangement. J.Combin.Theory Ser.A, 115:1156-1166, 2008.

A. van den Hombergh. About the automorphisms of the bruhat-ordering in a coxeter group. Indag. math, 36:125-131, 1974. 